\title{
INTEGRAL REPRESENTATIONS FOR THE JACOBI - PIÑEIRO POLYNOMIALS AND THE FUNCTIONS OF THE SECOND KIND
}

\begin{abstract}
We consider the Hermite-Padé approximants for the Cauchy transforms of the Jacobi weights in one interval. The denominators of the approximants are known as Jacobi-Piñeiro polynomials. These polynomials, together with the functions of the second kind, satisfy a generalized hypergeometric differential equation. In the case of the two weights, we construct the basis of the solutions of this ODE with elements of different growth rate. We obtain the integral representations for the basis elements.
\end{abstract}

Key words: Hermite-Padé approximants, Jacobi-Piñeiro multiple orthogonal polynomials, functions of the second kind, integral representations, generalized hypergeometric functions

2010 Mathematical Subject Classification: 33C20, 33C45

1. Introduction. We start from the definition of the Hermite-Padé approximants. Consider a vector $\vec{f}:=\left(f_{1}, \ldots, f_{r}\right)$ of germs of analytic functions in a neighborhood of infinity:

$$
f_{j}(z)=\sum_{k=0}^{\infty} \frac{c_{j, k}}{z^{k}}, \quad j=1, \ldots, r .
$$

Hermite-Padé approximants of type II for a vector $\vec{f}$ and a multi-index $\vec{n}:=\left(n_{1}, \ldots, n_{r}\right) \in \mathbb{Z}_{+}^{r}$ are the rational functions $\left(\frac{P_{\vec{n}, 1}}{Q_{\vec{n}}}, \ldots, \frac{P_{\vec{n}, r}}{Q_{\vec{n}}}\right)$, such that the degree of the denominator $Q_{\vec{n}}$ does not exceed $|\vec{n}|:=n_{1}+\cdots+n_{r}$ and the following interpolation conditions at infinity hold:

$$
R_{\vec{n}, j}(z):=\left(Q_{\vec{n}} f_{j}-P_{\vec{n}, j}\right)(z)=O\left(\frac{1}{z^{n_{j}+1}}\right), \quad z \rightarrow \infty, \quad j=1, \ldots, r .
$$

(C) Petrozavodsk State University, 2019 
The conditions (1) form a linear homogeneous system of $|\vec{n}|$ equations for $|\vec{n}|+1$ unknown coefficients of $Q_{\vec{n}}$. So, a nontrivial solution always exists. The numerator $P_{\vec{n}, j}$ is the polynomial part of the Laurent expansion of $Q_{\vec{n}} f_{j}$ at infinity. The remainder function $R_{\vec{n}, j}$ is the main part of the same Laurent expansion. It is called the function of the second kind.

In this paper, we consider the Hermite-Padé approximants for a set of Markov functions:

$$
f_{j}(z)=\widehat{w}_{j}(z):=\int_{\Delta} \frac{w_{j}(x) d x}{z-x}, \quad z \in \overline{\mathbb{C}} \backslash \Delta, \quad j=1, \ldots, r,
$$

where $\Delta:=[0,1]$ and $w_{1}, \ldots, w_{r}$ are the Jacobi positive weights in $(0,1)$ :

$$
w_{j}(x):=x^{\alpha_{j}}(1-x)^{\beta}, \quad x \in(0,1), \quad \alpha_{j}, \beta>-1, \quad j=1, \ldots, r .
$$

It is well known (see [10]), that for the Markov functions (2) the interpolation conditions (1) are equivalent to the system of orthogonality relations with respect to the weights $w_{j}$ :

$$
\int_{\Delta} Q_{\vec{n}}(x) x^{k} w_{j}(x) d x=0, \quad k=0, \ldots, n_{j}-1, \quad j=1, \ldots, r .
$$

The functions of the second kind can be represented as follows:

$$
R_{\vec{n}, j}(z)=\frac{1}{p_{n_{j}}(z)} \int_{\Delta} \frac{p_{n_{j}}(x) Q_{\vec{n}}(x) w_{j}(x) d x}{z-x} \quad \forall p_{n_{j}} \not \equiv 0: \operatorname{deg} p_{n_{j}} \leqslant n_{j} .
$$

Due to the orthogonality conditions (4), the left-hand side of (5) does not depend on the choice of the polynomial $p_{n_{j}}$ of degree not exceeding $n_{j}$.

It is natural to assume that the exponents $\alpha_{j}$ have pairwise different fractional parts: $\alpha_{j}-\alpha_{k} \notin \mathbb{Z}$ as $j \neq k$. Then the set of weights (3) forms an algebraic Chebyshev system (AT-system, see [10]) in $\Delta$. It follows that the polynomial $Q_{\vec{n}}$ has $|\vec{n}|$ simple zeros in $\Delta$. Thus, all the indices $\vec{n}$ are normal, i. e, the degree of $Q_{\vec{n}}$ is equal to $|\vec{n}|$, it is determined up to a constant factor, and the Hermite-Padé approximants are uniquely determined.

The Hermite-Padé approximants for the set (2), (3) were introduced in [11]. The polynomials $Q_{\vec{n}}$ are called the Jacobi-Piñeiro polynomials of multiple orthogonality [2]. Whenever it is necessary to emphasize the 
dependence on the parameters $\vec{\alpha}$ and $\beta$, the denominators $Q_{\vec{n}}$ and the functions of the second kind $R_{\vec{n}, j}$ will be also denoted by $Q_{\vec{n}}^{\vec{\alpha}, \beta}$ and $R_{\vec{n}, j}^{\vec{\alpha}, \beta}$. In the case $r=1, Q_{n}^{\alpha, \beta}$ is the classical Jacobi polynomial on $[0,1]$ and $R_{n}^{\alpha, \beta}$ is the corresponding function of the second kind, see [13], [9].

The Jacobi - Piñeiro polynomials have a number of applications. E. Mukhin and A. Varchenko [7] show that the zeros of $Q_{\vec{n}}$ along with the zeros of their Wronskian-type determinants are the unique solution of a certain Bethe Ansatz equation. C. Smet and W. Van Assche [12] show that their Mellin transforms can be applied to prove the Apéri theorem on the irrationality of $\zeta(3)$ and the Ball-Rivoal theorem on the infinite number of irrational points among $\{\zeta(2 m+1)\}$. M. Adler, P. van Moerbeke and D. Wang [1] relate the Jacobi-Piñeiro polynomials to certain problems of random matrix minor processes in the percolation theory.

The algebraic properties of $Q_{\vec{n}}$ were studied in [2], [4]. The limit zero distribution of $Q_{\vec{n}}$ was investigated in [8], the asymptotics near the edges of $\Delta$ was considered in [3]. The aim of this paper is to obtain integral representations, which can be used for describing the asymptotic behavior of $Q_{\vec{n}}$ and $R_{\vec{n}, j}$ in different asymptotic regimes that are important in applications.

The Jacobi - Piñeiro polynomials have an explicit representation [2,11]:

$$
(1-z)^{\beta} Q_{\vec{n}}(z)=\left(\prod_{j=1}^{r} \frac{z^{-\alpha_{j}}}{n_{j} !} \frac{d^{n_{j}}}{d z^{n_{j}}} z^{\alpha_{j}+n_{j}}\right)(1-z)^{\beta+|\vec{n}|} .
$$

The operators in the product of the formula (6) commute. The orthogonality relations (4) can be easily checked using the integration by parts. Similarly to the classical case $r=1$, we will call the representation (6) the Rodrigues formula. The formula (6) fixes a normalization of $Q_{\vec{n}}$ :

$$
Q_{\vec{n}}(0)=\prod_{j=1}^{r} \frac{\left(\alpha_{j}+1\right)_{n_{j}}}{n_{j} !}
$$

where $(\alpha)_{n}:=(\alpha) \cdots(\alpha+n-1)$ is the Pochhammer symbol. In what follows, we will use this normalization.

2. Generalized hypergeometric differential equation. The Jacobi-Piñeiro polynomials preserve many properties of classical Jacobi polynomials: they have Rodrigues representations, satisfy certain recurrence relations. Another such property is the relationship with (generali- 
zed) hypergeometric functions (see [4]):

$$
(1-z)^{\beta} \frac{Q_{\vec{n}}(z)}{Q_{\vec{n}}(0)}={ }_{r+1} F_{r}\left(\begin{array}{l}
\vec{\alpha}+\overrightarrow{1}+\vec{n},-\beta-|\vec{n}| \\
\vec{\alpha}+\overrightarrow{1}
\end{array} z\right)=: F_{0}(z),
$$

where $\overrightarrow{1}=(1, \ldots, 1)$ and

$$
{ }_{p} F_{q}\left(\begin{array}{l}
\vec{a} \\
\vec{b}
\end{array} ; z\right):=\sum_{k=0}^{\infty} \frac{\left(a_{1}\right)_{k} \ldots\left(a_{p}\right)_{k}}{\left(b_{1}\right)_{k} \ldots\left(b_{q}\right)_{k}} \frac{z^{k}}{k !} .
$$

It immediately follows from (7) that the function $y(z)=(1-z)^{\beta} Q_{\vec{n}}(z)$ satisfies the linear ordinary differential equation of order $r+1$ :

$$
\begin{aligned}
L_{z} y=0, \quad L_{z}:=\left(z \frac{d}{d z}-\beta-|\vec{n}|\right) \prod_{j=1}^{r}\left(z \frac{z}{d z}\right. & \left.+\alpha_{j}+1+n_{j}\right)- \\
& -\frac{d}{d z} \prod_{j=1}^{r}\left(z \frac{z}{d z}+\alpha_{j}\right) .
\end{aligned}
$$

The set of functions

$$
F_{0}, z^{-\alpha_{1}} F_{1}, \ldots, z^{-\alpha_{r}} F_{r}
$$

forms a basis of the solutions of (8), where

$$
F_{j}(z):={ }_{r+1} F_{r}\left(\begin{array}{ll}
\vec{\alpha}+\vec{n}+\left(1-\alpha_{j}\right) \overrightarrow{1}, & -\beta-\alpha_{j}-|\vec{n}| \\
{[\vec{\alpha}]_{j}+\left(1-\alpha_{j}\right)[\overrightarrow{1}]_{j},} & 1-\alpha_{j}
\end{array} ;\right)
$$

and $[\vec{\alpha}]_{j}:=\left(\alpha_{1}, \ldots, \alpha_{j-1}, \alpha_{j+1}, \ldots, \alpha_{r}\right)$ is the projection of $\vec{\alpha}$ onto the hyperspace orthogonal to $\vec{e}_{j}$.

On the other hand, it can be shown that for some constants $b_{j} \neq 0$ we have

$$
\frac{R_{\vec{n}, j}(z)}{Q_{\vec{n}}(0)}=\frac{\pi}{\sin \pi \alpha_{j}}(-z)^{\alpha_{j}} F_{0}(z)+b_{j} F_{j}(z) .
$$

Therefore,

$$
(1-z)^{\beta} Q_{\vec{n}}, z^{-\alpha_{1}} R_{\vec{n}, 1}, \ldots, z^{-\alpha_{r}} R_{\vec{n}, r}
$$

is another basis in the space of solutions of (8).

3. Integral representations. Further, for the sake of simplicity, we restrict ourselves to the case of $r=2$ weights. 
For large values of $|\vec{n}|$, the basis (10) is preferable to the basis (9), since only the first element of (10) has the maximum growth rate. It would be helpful to construct a basis where all the elements have pairwise different growth rates. Nonetheless, it turns out that for $\alpha_{1}-\alpha_{2} \in(0,1)$ and for the diagonal indices $n_{1}=n_{2}$ the functions $z^{-\alpha_{1}} R_{\vec{n}, 1}$ and $z^{-\alpha_{2}} R_{\vec{n}, 2}$ are asymptotically close. Indeed, in [6] it is shown that $\frac{R_{\vec{n}, 2}}{R_{\vec{n}, 1}} \rightarrow z^{\alpha_{2}-\alpha_{1}}$ for $z \in \mathbb{C} \backslash(-\infty, 1]$ as $n_{1}=n_{2} \rightarrow \infty$, where $z^{\alpha_{2}-\alpha_{1}}$ is analytic outside $(-\infty, 0]$ and positive for $z>0$.

Therefore, along with the functions $R_{\vec{n}, j}$, we consider the functions $R_{\vec{n}, j}^{*}($ see $[5])$ :

$$
R_{\vec{n}, 2}^{*}:=-z^{\alpha_{2}-\alpha_{1}} R_{\vec{n}, 1}^{*}:=z^{\alpha_{2}-\alpha_{1}} R_{\vec{n}, 1}-R_{\vec{n}, 2} .
$$

Thus, we establish that

$$
(1-z)^{\beta} Q_{\vec{n}}, z^{-\alpha_{1}} R_{\vec{n}, 1}, z^{-\alpha_{1}} R_{\vec{n}, 1}^{*}
$$

is a basis of the solutions of (8) for $r=2$.

It directly follows from the definition (11) that the functions $R_{\vec{n}, j}^{*}$ are analytic outside $(-\infty, 1]$. Applying the Sokhotski-Plemelj formula to (5), we calculate the jumps of $R_{\vec{n}, j}$ in the interval $(0,1)$ :

$$
R_{\vec{n}, j}(x+i 0)-R_{\vec{n}, j}(x-i 0)=-2 \pi i Q_{\vec{n}}(x) w_{j}(x), \quad x \in(0,1) .
$$

Clearly, the jumps of $R_{\vec{n}, j}^{*}$ in $(0,1)$ are equal to zero and the singularities at the point 1 are removable. So, in fact, $R_{\vec{n}, j}^{*}$ are analytic outside $(-\infty, 0]$.

For $\alpha \in(0,1)$ the following representation for $z^{-\alpha}$ holds:

$$
z^{-\alpha}=\frac{\sin \pi \alpha}{\pi} \int_{-\infty}^{0} \frac{(-x)^{-\alpha} d x}{z-x}, \quad z \in \mathbb{C} \backslash(-\infty, 0] .
$$

So for $\alpha_{1}-\alpha_{2} \in(0,1)$ we have the following integral representation for the function $R_{\vec{n}, 2}^{*}$ :

$$
R_{\vec{n}, 2}^{*}(z)=\frac{\sin \pi\left(\alpha_{1}-\alpha_{2}\right)}{\pi} \int_{-\infty}^{0} \frac{R_{\vec{n}, 1}(x)}{z-x}(-x)^{\alpha_{2}-\alpha_{1}} d x .
$$

The formula (13) carries out an analytic continuation of $R_{\vec{n}, 2}^{*}$ from the domain $\mathbb{C} \backslash(-\infty, 1]$ to $\mathbb{C} \backslash(-\infty, 0]$. 
We introduce the notation for three double integrals:

$$
\begin{aligned}
& F_{\vec{n}, 0}(z):=\oint_{\gamma_{z}} \frac{t^{n_{1}+\alpha_{1}-\alpha_{2}} d t}{(t-z)^{n_{1}+1}} \oint_{\gamma_{t}} \frac{s^{n_{2}+\alpha_{2}}(1-s)^{n_{1}+n_{2}+\beta} d s}{(s-t)^{n_{2}+1}}, \\
& F_{\vec{n}, 1}(z):=\int_{0}^{1} \frac{t^{n_{1}+\alpha_{1}-\alpha_{2}} d t}{(t-z)^{n_{1}+1}} \oint_{\gamma_{t}} \frac{s^{n_{2}+\alpha_{2}}(1-s)^{n_{1}+n_{2}+\beta} d s}{(s-t)^{n_{2}+1}}, \\
& F_{\vec{n}, 1}^{*}(z):=\int_{-\infty}^{0} \frac{t^{n_{1}}(-t)^{\alpha_{1}-\alpha_{2}} d t}{(t-z)^{n_{1}+1}} \int_{0}^{1} \frac{s^{n_{2}+\alpha_{2}}(1-s)^{n_{1}+n_{2}+\beta} d s}{(s-t)^{n_{2}+1}},
\end{aligned}
$$

where $\gamma_{z}, \gamma_{t}$ are small enough positively oriented circles around the points $z$ and $t$, respectively. The first two integrals absolutely converge under standard conditions on the parameters: $\alpha_{1}, \alpha_{2}, \beta>-1, n_{1}, n_{2} \in \mathbb{Z}_{+}$. For absolute convergence of the third integral, we additionally require that $\alpha_{1}-\alpha_{2} \in\left(-n_{1}-1, n_{2}+1\right)$. Under these conditions, these integrals determine holomorphic functions in the following domains:

$$
F_{\vec{n}, 0} \in H(\mathbb{C} \backslash \overline{\mathbb{R} \backslash \Delta}), \quad F_{\vec{n}, 1} \in H(\mathbb{C} \backslash \Delta), \quad F_{\vec{n}, 1}^{*} \in H(\mathbb{C} \backslash(-\infty, 0]) .
$$

The relation between these integrals and the considered Hermite-Padé approximants is given in the following theorem.

Theorem 1. We have the following integral representations for the set of functions $\left(w_{1} Q_{\vec{n}}, R_{\vec{n}, 1}, R_{\vec{n}, 1}^{*}\right)$ :

$$
\left(w_{1} Q_{\vec{n}}, R_{\vec{n}, 1}, R_{\vec{n}, 1}^{*}\right)=-\left(\frac{F_{\vec{n}, 0}}{4 \pi^{2}}, \frac{F_{\vec{n}, 1}}{2 \pi i}, \frac{\sin \pi\left(\alpha_{1}-\alpha_{2}\right) F_{\vec{n}, 1}^{*}}{\pi}\right) .
$$

The integral representations for $Q_{\vec{n}}, R_{\vec{n}, 1}$ are simple consequences of the Rodrigues formula (6), the formula for $R_{\vec{n}, 1}^{*}$ is less trivial.

4. Integral representation for $Q_{\vec{n}}$. Rewrite the Rodrigues formula (6) for the case $r=2$ :

$$
z^{\alpha_{1}}(1-z)^{\beta} Q_{\vec{n}}(z)=\frac{1}{n_{1} !} \frac{d^{n_{1}}}{d z^{n_{1}}} z^{n_{1}+\alpha_{1}-\alpha_{2}} \frac{1}{n_{2} !} \frac{d^{n_{2}}}{d z^{n_{2}}} z^{n_{2}+\alpha_{2}}(1-z)^{n_{1}+n_{2}+\beta} .
$$

We obtain the integral representation for $Q_{\vec{n}}$ from this formula by applying twice the Cauchy formula for derivative of an analytic function. Indeed, according to the Cauchy formula, we have 


$$
\begin{aligned}
& \frac{1}{n_{2} !} \frac{d^{n_{2}}}{d t^{n_{2}}} t^{n_{2}+\alpha_{2}}(1-t)^{n_{1}+n_{2}+\beta}= \\
&=\frac{1}{2 \pi i} \oint_{\gamma_{t}} \frac{s^{n_{2}+\alpha_{2}}(1-s)^{n_{1}+n_{2}+\beta} d s}{(s-t)^{n_{2}+1}}=: \frac{G(t)}{2 \pi i} .
\end{aligned}
$$

By definition, the functions $F_{\vec{n}, 0}, F_{\vec{n}, 1}$ are related to $G$ by the equalities:

$$
F_{\vec{n}, 0}(z)=\oint_{\gamma_{z}} \frac{t^{n_{1}+\alpha_{1}-\alpha_{2}} G(t) d t}{(t-z)^{n_{1}+1}}, \quad F_{\vec{n}, 1}(z)=\int_{0}^{1} \frac{t^{n_{1}+\alpha_{1}-\alpha_{2}} G(t) d t}{(t-z)^{n_{1}+1}} .
$$

Applying the Cauchy formula again, we obtain the required:

$$
w_{1}(z) Q_{\vec{n}}(z)=\frac{1}{2 \pi i} \frac{1}{n_{1} !} \frac{d^{n_{1}}}{d z^{n_{1}}} z^{n_{1}+\alpha_{1}-\alpha_{2}} G(z)=\frac{1}{(2 \pi i)^{2}} \oint_{\gamma_{z}} \frac{t^{n_{1}+\alpha_{1}-\alpha_{2}} G(t) d t}{(t-z)^{n_{1}+1}} .
$$

Note that by the formula (6) (for $r=1$ ) the function $G$ defined by the formula (15) is expressed through the classical Jacobi polynomials:

$$
\frac{G(t)}{2 \pi i}=t^{\alpha_{2}}(1-t)^{n_{1}+\beta} Q_{n_{2}}^{\alpha_{2}, n_{1}+\beta}(t) .
$$

5. Integral representation for $R_{\vec{n}, 1}$. We need the following lemma, which is a consequence of the partial integration formula.

Lemma 1. 1) Let $\widetilde{u}, \widetilde{v} \in C^{n}[0,1]$ and $u(x):=x^{c_{0}}(1-x)^{c_{1}} \widetilde{u}(x)$, $v(x):=x^{d_{0}}(1-x)^{d_{1}} \widetilde{v}(x)$, where $c_{0}+d_{0}>n-1$ and $c_{1}+d_{1}>n-1$; then

$$
\int_{0}^{1} u(x) \frac{d^{n} v}{d x^{n}}(x) d x=(-1)^{n} \int_{0}^{1} \frac{d^{n} u}{d x^{n}}(x) v(x) d x .
$$

2) Let $\widetilde{u}, \widetilde{v} \in C^{n}[1,+\infty), \widetilde{u}^{(k)}(x), \widetilde{v}^{(k)}(x)=O\left(x^{-k}\right)$ as $x \rightarrow+\infty, k=$ $=0, \ldots, n$ and $u(x):=x^{c_{0}-c_{1}}(x-1)^{c_{1}} \widetilde{u}(x), v(x):=x^{d_{0}-d_{1}}(x-1)^{d_{1}} \widetilde{v}(x)$, where $c_{1}+d_{1}>n-1$ and $c_{0}+d_{0}<n-1$; then

$$
\int_{1}^{+\infty} u(x) \frac{d^{n} v}{d x^{n}}(x) d x=(-1)^{n} \int_{1}^{+\infty} \frac{d^{n} u}{d x^{n}}(x) v(x) d x
$$


The conditions of the lemma are sufficient for the absolute convergence of the integrals and equality to zero of all non-integral terms, see section 7 for the proof.

Integral representation for $R_{\vec{n}, 1}$ is derived from (5) and (16) applying Lemma 1 and (17):

$$
\begin{aligned}
R_{\vec{n}, 1}(z) & =\int_{0}^{1} \frac{Q_{\vec{n}}(t) w_{1}(t) d t}{z-t}= \\
= & \frac{1}{2 \pi i} \int_{0}^{1} \frac{d t}{z-t} \frac{1}{n_{1} !} \frac{d^{n_{1}}}{d t^{n_{1}}} t^{n_{1}+\alpha_{1}-\alpha_{2}} G(t)=\frac{-1}{2 \pi i} \int_{0}^{1} \frac{t^{n_{1}+\alpha_{1}-\alpha_{2}} G(t) d t}{(t-z)^{n_{1}+1}} .
\end{aligned}
$$

We obtain one more integral representation for $R_{\vec{n}, 1}$. We use the formula (15) and again apply Lemma 1 to continue the chain of identities:

$$
\begin{aligned}
& -R_{\vec{n}, 1}(z)=\int_{0}^{1} \frac{t^{n_{1}+\alpha_{1}-\alpha_{2}} d t}{(t-z)^{n_{1}+1}} \frac{1}{n_{2} !} \frac{d^{n_{2}}}{d t^{n_{2}}} t^{n_{2}+\alpha_{2}}(1-t)^{n_{1}+n_{2}+\beta}= \\
& =(-1)^{n_{2}} \int_{0}^{1} t^{n_{2}+\alpha_{2}}(1-t)^{n_{1}+n_{2}+\beta} d t \frac{1}{n_{2} !} \frac{d^{n_{2}}}{d t^{n_{2}}} \frac{t^{n_{1}+\alpha_{1}-\alpha_{2}}}{(t-z)^{n_{1}+1}}= \\
& =\frac{(-1)^{n_{1}+n_{2}+1}}{z^{n_{2}+1-\alpha_{1}+\alpha_{2}}} \int_{0}^{1} t^{n_{2}+\alpha_{2}}(1-t)^{n_{1}+n_{2}+\beta} d t \frac{1}{n_{2} !} \frac{d^{n_{2}}}{d(t / z)^{n_{2}}} \frac{\left(\frac{t}{z}\right)^{n_{1}+\alpha_{1}-\alpha_{2}}}{\left(1-\frac{t}{z}\right)^{n_{1}+1}} .
\end{aligned}
$$

Denote by $q_{\vec{n}}$ a polynomial of degree $n_{1}$ defined by the formula:

$$
q_{\vec{n}}(x):=\frac{(1-x)^{n_{1}+n_{2}+1}}{x^{\alpha_{1}-\alpha_{2}} n_{2} !} \frac{d^{n_{2}}}{d x^{n_{2}}} \frac{x^{n_{1}+\alpha_{1}-\alpha_{2}}}{(1-x)^{n_{1}+1}} .
$$

Then we obtain the following relation for $R_{\vec{n}, 1}$ :

$$
-R_{\vec{n}, 1}(z)=\int_{0}^{1} t^{n_{2}+\alpha_{1}}(1-t)^{n_{1}+n_{2}+\beta} \frac{z^{n_{1}} q_{\vec{n}}\left(\frac{t}{z}\right) d t}{(t-z)^{n_{1}+n_{2}+1}} .
$$

6. Integral representation for $R_{\vec{n}, 1}^{*}$. We now turn to the proof of the last relation (14). The definition of $R_{\vec{n}, j}^{*}$ (see (11)) implies that $R_{\vec{n}, 1}^{*}=-\frac{w_{1}}{w_{2}} R_{\vec{n}, 2}^{*}$. We will use the representation (13), which is valid under the condition $\alpha_{1}-\alpha_{2} \in(0,1)$. First we prove what is required under this 
restriction, and then remove the restriction by applying the principle of analytic continuation on $\vec{\alpha}$. So, we have the following chain of equalities:

$$
\begin{gathered}
\frac{\pi R_{\vec{n}, 2}^{*}(z)}{\sin \pi\left(\alpha_{1}-\alpha_{2}\right)}=\int_{-\infty}^{0} \frac{R_{\vec{n}, 1}(x)(-x)^{\alpha_{2}-\alpha_{1}} d x}{z-x}= \\
=\int_{-\infty}^{0} \frac{(-x)^{\alpha_{2}-\alpha_{1}} d x}{x-z} \int_{0}^{1} s^{n_{2}+\alpha_{1}}(1-s)^{n_{1}+n_{2}+\beta} \frac{x^{n_{1}} q_{\vec{n}}\left(\frac{s}{x}\right) d s}{(s-x)^{n_{1}+n_{2}+1}}= \\
=\int_{0}^{1} s^{n_{2}+\alpha_{1}}(1-s)^{n_{1}+n_{2}+\beta} d s \int_{-\infty}^{0} \frac{x^{n_{1}} q_{\vec{n}}\left(\frac{s}{x}\right)(-x)^{\alpha_{2}-\alpha_{1}} d x}{(s-x)^{n_{1}+n_{2}+1}(x-z)}= \\
=\int_{0}^{1} s^{n_{2}+\alpha_{1}}(1-s)^{n_{1}+n_{2}+\beta} I(s, z) d s,
\end{gathered}
$$

where

$$
I(s, z):=\int_{-\infty}^{0} \frac{x^{n_{1}} q_{\vec{n}}\left(\frac{s}{x}\right)(-x)^{\alpha_{2}-\alpha_{1}} d x}{(s-x)^{n_{1}+n_{2}+1}(x-z)}, \quad s>0, z \in \mathbb{C} \backslash(-\infty, 0] .
$$

Let us transform the integral $I(s, z)$. First, we change the variable $x$ to $\tau=s / x$ :

$$
\begin{gathered}
I(s, z)=\int_{-\infty}^{0} \frac{\left(\frac{s}{\tau}\right)^{n_{1}} q_{\vec{n}}(\tau)\left(\frac{s}{-\tau}\right)^{\alpha_{2}-\alpha_{1}} \frac{s}{\tau^{2}} d \tau}{\left(s-\frac{s}{\tau}\right)^{n_{1}+n_{2}+1}\left(\frac{s}{\tau}-z\right)}= \\
=\frac{(-1)^{n_{1}+n_{2}+1}}{z s^{n_{2}+\alpha_{1}-\alpha_{2}}} \int_{-\infty}^{0} \frac{\tau^{n_{2}} q_{\vec{n}}(\tau)(-\tau)^{\alpha_{1}-\alpha_{2}} d \tau}{(1-\tau)^{n_{1}+n_{2}+1}\left(\frac{s}{z}-\tau\right)} .
\end{gathered}
$$

The expression (18) for the polynomial $q_{\vec{n}}$ can be rewritten, changing the branch of the power function in the numerator and denominator:

$$
q_{\vec{n}}(x)=\frac{(1-x)^{n_{1}+n_{2}+1}}{(-x)^{\alpha_{1}-\alpha_{2}} n_{2} !} \frac{d^{n_{2}}}{d x^{n_{2}}} \frac{x^{n_{1}}(-x)^{\alpha_{1}-\alpha_{2}}}{(1-x)^{n_{1}+1}} .
$$

We substitute this expression for $q_{\vec{n}}$ in the integral and again apply Lemma 1 (recall that for now $\alpha_{1}-\alpha_{2} \in(0,1)$ ): 


$$
\begin{gathered}
I(s, z)=\frac{(-1)^{n_{1}+n_{2}+1}}{z s^{n_{2}+\alpha_{1}-\alpha_{2}}} \int_{-\infty}^{0} \frac{\tau^{n_{2}} d \tau}{\left(\frac{s}{z}-\tau\right)} \frac{1}{n_{2} !} \frac{d^{n_{2}}}{d \tau^{n_{2}}} \frac{\tau^{n_{1}}(-\tau)^{\alpha_{1}-\alpha_{2}}}{(1-\tau)^{n_{1}+1}}= \\
=\frac{(-1)^{n_{1}+1}}{z s^{n_{2}+\alpha_{1}-\alpha_{2}}} \int_{-\infty}^{0} \frac{\tau^{n_{1}}(-\tau)^{\alpha_{1}-\alpha_{2}} d \tau}{(1-\tau)^{n_{1}+1}} \frac{1}{n_{2} !} \frac{d^{n_{2}}}{d \tau^{n_{2}}} \frac{\tau^{n_{2}}}{\left(\frac{s}{z}-\tau\right)}
\end{gathered}
$$

Note that the following identity holds:

$$
\frac{1}{n_{2} !} \frac{d^{n_{2}}}{d \tau^{n_{2}}} \frac{\tau^{n_{2}}}{\left(\frac{s}{z}-\tau\right)}=\frac{\left(\frac{s}{z}\right)^{n_{2}}}{\left(\frac{s}{z}-\tau\right)^{n_{2}+1}} .
$$

Using this equality, we remove one floor in the formula for $I(s, z)$. Then the substitution $\tau=\frac{t}{z}$ gives:

$$
\begin{gathered}
I(s, z)=\frac{(-1)^{n_{1}+1}}{z s^{n_{2}+\alpha_{1}-\alpha_{2}}} \int_{-\infty}^{0} \frac{\tau^{n_{1}}(-\tau)^{\alpha_{1}-\alpha_{2}}}{(1-\tau)^{n_{1}+1}} \frac{\left(\frac{s}{z}\right)^{n_{2}} d \tau}{\left(\frac{s}{z}-\tau\right)^{n_{2}+1}}= \\
=(z s)^{\alpha_{2}-\alpha_{1}} \int_{z \mathbb{R}_{-}} \frac{t^{n_{1}}(-t)^{\alpha_{1}-\alpha_{2}} d t}{(t-z)^{n_{1}+1}(s-t)^{n_{2}+1}}
\end{gathered}
$$

In the last integral, we deform the contour by replacing the ray $z \mathbb{R}_{-}$with $\mathbb{R}_{-}$. This can be done by the Cauchy theorem, since the singularities of the integrand at the points $s$ and $z$ are in one of the two angles formed by the rays, and it has the following behavior in a neighborhood of infinity: $O\left(1 / t^{n_{2}+2+\alpha_{2}-\alpha_{1}}\right)=o(1 / t)$. We get the following expression for $I(s, z)$ :

$$
I(s, z)=(z s)^{\alpha_{2}-\alpha_{1}} \int_{-\infty}^{0} \frac{t^{n_{1}}(-t)^{\alpha_{1}-\alpha_{2}} d t}{(t-z)^{n_{1}+1}(s-t)^{n_{2}+1}} .
$$

Substituting this formula in (20), we obtain the expression for $R_{\vec{n}, 2}^{*}$ matching the required after changing the order of integration:

$$
\begin{aligned}
& \frac{-\pi R_{\vec{n}, 1}^{*}(z)}{\sin \pi\left(\alpha_{1}-\alpha_{2}\right)}=\frac{\pi R_{\vec{n}, 2}^{*}(z) z^{\alpha_{1}-\alpha_{2}}}{\sin \pi\left(\alpha_{1}-\alpha_{2}\right)}= \\
& \quad=\int_{0}^{1} s^{n_{2}+\alpha_{2}}(1-s)^{n_{1}+n_{2}+\beta} d s \int_{-\infty}^{0} \frac{t^{n_{1}}(-t)^{\alpha_{1}-\alpha_{2}} d t}{(t-z)^{n_{1}+1}(s-t)^{n_{2}+1}}=F_{\vec{n}, 1}^{*}(z) .
\end{aligned}
$$


Thus, the following implication is proved:

$$
\alpha_{1}-\alpha_{2} \in(0,1) \quad \Longrightarrow \quad R_{\vec{n}, 1}^{*}(z)=\frac{\sin \pi\left(\alpha_{1}-\alpha_{2}\right)}{\pi} F_{\vec{n}, 1}^{*}(z) .
$$

Let us note that in the above identity the left-hand side $R_{\vec{n}, 1}^{*}:=\frac{w_{1}}{w_{2}} R_{\vec{n}, 2}-R_{\vec{n}, 1}$ analytically depends on $\vec{\alpha}$ in the domain $\left\{\vec{\alpha}: \operatorname{Re} \alpha_{j}>-1, j=1,2\right\}$ by virtue of the proved integral representations for $R_{\vec{n}, j}$ (the integral for $R_{\vec{n}, 2}$ is obtained from the integral for $R_{\vec{n}, 1}$ by permutation of indices $1 \leftrightarrow 2$ ). The integral $F_{\vec{n}, 1}^{*}$ in the right-hand side of this equality is an analytical function in $\left\{\vec{\alpha}: \operatorname{Re}\left(\alpha_{1}-\alpha_{2}\right) \in\left(-n_{1}-1, n_{2}+1\right)\right.$, $\left.\operatorname{Re} \alpha_{j}>-1, j=1,2\right\}$. By the uniqueness theorem, the equality holds in the whole domain.

Note that for the function $F_{\vec{n}, 1}^{*}$, the analogue of representation (16) holds:

$$
F_{\vec{n}, 1}^{*}(z)=\int_{-\infty}^{0} \frac{t^{n_{1}}(-t)^{\alpha_{1}-\alpha_{2}} g(t) d t}{(t-z)^{n_{1}+1}}
$$

where $g$ is the function of the second kind for the classical Jacobi polynomials (compare with the expression (17) for $G$ ):

$$
g(t):=\int_{0}^{1} \frac{s^{n_{2}+\alpha_{2}}(1-s)^{n_{1}+n_{2}+\beta} d s}{(s-t)^{n_{2}+1}}=\frac{-\widehat{G}(t)}{2 \pi i}=-R_{n_{2}}^{\alpha_{2}, n_{1}+\beta}(t) .
$$

7. Proof of Lemma 1. 1. It is enough to verify the convergence of at least one of the integrals and equality to zero of all non-integral terms in the formula of integration by parts:

$$
\int_{0}^{1}\left(u v^{(n)}\right)(x) d x=\left.\sum_{k=0}^{n-1}(-1)^{k}\left(u^{(k)} v^{(n-1-k)}\right)\right|_{0} ^{1}+(-1)^{n} \int_{0}^{1}\left(u^{(n)} v\right)(x) d x .
$$

The singularities at the ends are integrable, because

$$
\left(u^{(n)} v\right)(x)=x^{c_{0}+d_{0}-n}(1-x)^{c_{1}+d_{1}-n} \widetilde{u}_{n}(x)
$$

for certain function $\widetilde{u}_{n} \in C[0,1]$ and $c_{j}+d_{j}-n>-1, j=0,1$. All the non-integral terms equal zero, because

$$
\left(u^{(k)} v^{(n-1-k)}\right)(x)=x^{c_{0}+d_{0}-n+1}(1-x)^{c_{1}+d_{1}-n+1} \widetilde{u}_{k}(x)
$$


for certain function $\widetilde{u}_{k} \in C^{\min (k+1, n-k)}[0,1]$, where $k=0, \ldots, n-1$.

2. We apply the formula of integration by parts. Similarly to the previous case, we verify that the integrals converge at point 1 and all the non-integral terms at this point are equal to zero. We only need to verify the convergence and vanishing of the non-integral terms at infinity. By the Leibniz formula, it is easy to see that for $k=0, \ldots, n$ we have

$$
\frac{d^{k}}{d x^{k}}\left(1-\frac{1}{x}\right)^{c_{1}} \widetilde{u}(x)=O\left(x^{-k}\right), \quad x \rightarrow+\infty .
$$

By the Leibniz formula for the $k$ th derivative of the product:

$$
u^{(k)}(x)=x^{c_{0}-k} \sum_{j=0}^{k} b_{k, j} x^{j} \frac{d^{j}}{d x^{j}}\left(1-\frac{1}{x}\right)^{c_{1}} \widetilde{u}(x)=O\left(x^{c_{0}-k}\right), \quad x \rightarrow+\infty,
$$

where $b_{k, j}:=\left(\begin{array}{c}k \\ j\end{array}\right)(k-j) !\left(\begin{array}{c}c_{0} \\ k-j\end{array}\right)$ are constants. Similar relations hold for the function $v$. Eventually, we get that the function $u^{(n)} v$ is integrable at infinity, because $\left(u^{(n)} v\right)(x)=O\left(x^{c_{0}+d_{0}-n}\right)=O\left(x^{-1-\varepsilon}\right)$ as $x \rightarrow+\infty$, where $\varepsilon:=n-1-c_{0}-d_{0}>0$ and all the non-integral terms vanish at infinity:

$$
\left(u^{(k)} v^{(n-1-k)}\right)(x)=O\left(x^{-\varepsilon}\right), \quad x \rightarrow+\infty, \quad k=0, \ldots, n-1 .
$$

\section{References}

[1] Adler M., van Moerbeke P., Wang D. Random matrix minor processes related to percolation theory. Random Matrices Theory Appl., 2013, vol. 2, no. 4, 72 pp. DOI: https://doi.org/10.1142/S2010326313500081

[2] Aptekarev A. I., Branquinho A., Van Assche W. Multiple Orthogonal Polynomials for Classical Weights. Transactions of the American Mathematical Society, 2003, vol. 355, no. 10, pp. 3887-3914.

DOI: https://doi.org/10.1090/S0002-9947-03-03330-0

[3] Van Assche W. Mehler-Heine asymptotics for multiple orthogonal polynomials. Proc. Amer. Math. Soc., 2017, vol. 145 no. 1, pp. 303-314.

DOI: https://doi.org/10.1090/proc/13214

[4] Beckermann B., Coussement J., Van Assche W. Multiple Wilson and Jacobi-Pineiro polynomials. J. Approx. Theory, 2005, vol. 132, no. 2, pp. 155-181. DOI: https://doi.org/10.1016/j.jat.2004.12.001

[5] Gonchar A. A., Rakhmanov E. A., Sorokin V. N. Hermite-Padé approximants for systems of Markov type functions. Sb. Math., 1997, vol. 188, no. 5, pp. 671-696. DOI: https://doi.org/10.4213/sm225 
[6] Lysov V. G. Asymptotics of Jacobi-Piñeiro polynomials and functions of the second kind. Math. Notes, 2018, vol. 103, no. 3, pp. 495-498.

DOI: https://doi.org/10.4213/mzm11826

[7] Mukhin E. Varchenko A. Multiple orthogonal polynomials and a counterexample to the Gaudin Bethe ansatz conjecture. Trans. Amer. Math. Soc., 2007, vol. 359 , no. 11 , pp. $5383-5418$.

DOI: https://doi.org/10.1090/S0002-9947-07-04217-1

[8] Neuschel, T.; Van Assche, W. Asymptotic zero distribution of JacobiPineiro and multiple Laguerre polynomials. J. Approx. Theory, 2016, vol. 205, pp. $114-132$.

DOI: https://doi.org/10.1016/j.jat.2016.02.002

[9] Nikiforov A. F., Uvarov V. B. Special functions of mathematical physics: A unified introduction with applications. Birkhäuser, Boston, 1988.

[10] Nikishin E. M., Sorokin V. N. Rational Approximation and Orthogonality. Math. Monographs, vol. 92, Amer. Math. Soc., Providence, RI, 1991.

[11] Piñeiro Dias L. R. On simultaneous Padé approximants for a collection of Markov functions, Vestnik Moskov. Univ. Ser. I, 1987, no. 2, pp. 67-70.

[12] Smet C., Van Assche W. Mellin transforms for multiple Jacobi-Pineiro polynomials, J. Approx. Theory, 2010, vol. 162, pp. 782-806.

DOI: https://doi.org/10.1016/j.jat.2009.09.004

[13] Szegö G. Orthogonal Polynomials, American Mathematical Society, 1975.

Received August 16, 2019.

In revised form, October 29, 2019.

Accepted October 30, 2019.

Published online November 5, 2019.

Keldysh Institute of Applied Mathemtics, RAS

4 Miusskaya sq., Moscow 125047, Russia

E-mail: v.g.lysov@gmail.com 